\title{
A Pessoa Estomizada: Análise das Práticas Educativas de Enfermagem
}

\author{
The Ostomized People: Analysis of Educational Nursing Practices
}

La Persona Portadora de Ostomía: Análisis de las Prácticas Educativas de Enfermería

Bruna Gomes ${ }^{1}$, Shirley Santos Martins²

\begin{abstract}
RESUMO
Trata-se de um estudo descritivo tipo revisão integrativa com o objetivo de analisar as evidências disponíveis quanto às orientações de enfermagem fornecidas a pessoa estomizada. Coleta de dados de junho a julho de 2014, artigos publicados nos últimos 10 anos. Critérios de inclusão: Métodos explícitos, em português, adultos e idosos de ambos os sexos, periódicos on-line gratuitos a partir da busca avançada. Critérios de exclusão: estudos duplicados e não ter aderência ao objetivo do estudo. Obteve-se uma amostra de 10 artigos. As publicações se concentram no período de 2009 a 2010 (50\%). A literatura preconiza o inicio das orientações de enfermagem no pré-operatório. O conteúdo das orientações é variável entre os artigos não havendo nenhuma padronização/preconização específica de consensos e diretrizes para o assunto a ser abordado. Concluímos que há imensa lacuna no processo de reabilitação da pessoa com estoma e que podem ser solucionados com resgate das orientações fornecidas à clientela em termos de apreensão das informações.
\end{abstract}

DESCRITORES: Estomaterapia. Estomia. Educação em saúde. Enfermagem.

\begin{abstract}
This is a descriptive type integrative review study with the objective evidence available about the nursing instructions provided to ostomized people. Data collection from June to July 2014, articles published in the last 10 years. Inclusion criteria: Explicit methods, in Portuguese, adults and seniors of both sexes, online journals free from the advanced search. Exclusion criteria: duplicate studies and not adherence to the purpose of the study. There was obtained a sample of 10 items. The publications focus on the period 2009 to 2010 (50\%). The literature recommends the beginning of nursing instructions preoperatively. The content of the guidelines is variable between the articles and there is no standardization / preconization specific consensus and guidelines for the issue to be addressed. We conclude that there is huge gap in the person's rehabilitation process with stoma and can be solved with redemption of the guidance provided to customers in terms of seizure of information.
\end{abstract}

DESCRIPTORS: Stomatherapy. Ostomy. Health education. Nursing.

\footnotetext{
${ }^{1}$ Hospital Universitário Unidade Presidente Dutra (HUUPD) - São Luís (MA), Brasil. Endereço para correspondência: Bruna Gomes - Rua da Felicidade, 4 CEP: 65080-800 - São Luís (MA), Brasil. E-mail: brunagomes2000@yahoo.com.br

${ }^{2}$ Hospital Universitário da Universidade Federal do Maranhão - São Luís (MA), Brasil.

Artigo recebido em: 12/08/2015 - Aceito para publicação em: 26/03/2016
} 


\section{RESUMEN}

Este es un estudio de revisión integradora de tipo descriptivo con la evidencia objetiva disponible acerca de las instrucciones de enfermería prestados a las personas portadoras de ostomías. La recolección de datos de junio a julio de 2014, los artículos publicados en los últimos 10 años. Criterios de inclusión: métodos explícitos, en portugués, adultos y ancianos de ambos sexos, los diarios gratuitos en línea de la búsqueda avanzada. Criterios de exclusión: estudios duplicados y no adherencia a la finalidad del estudio. Se obtuvo una muestra de 10 artículos. Las publicaciones se centran en el período 2009 a 2010 (50\%). La literatura recomienda el inicio de las instrucciones de enfermería en el preoperatorio. El contenido de las directrices es variable entre los artículos y no hay una estandarización / consenso específico preconización y lineamientos para el tema a tratar. Llegamos a la conclusión de que existe una gran brecha en el proceso de rehabilitación de la persona con estoma y puede ser resuelto con la redención de la orientación proporcionada a los clientes en términos de incautación de información.

DESCRIPTORES: Estomaterapia. Estomía. Educación en salud. Enfermería.

\section{INTRODUÇÃO}

Estoma e estomia são palavras de origem grega que significam boca ou abertura, utilizada para indicar a exteriorização de qualquer víscera oca através do corpo por causas variadas, desviando o transito normal ${ }^{1}$. A terminologia médica apresenta numerosos casos de impropriedades evitáveis, como exemplo o termo grego stoma, na transmudação dos termos gregos para o português e suas formas derivadas, com algumas exceções. $\mathrm{Na}$ formação de nomes com elementos procedentes do grego ou do latim, usa-se o $e$ antes de termos iniciados por “s”, e não “o”, como exemplo, dessa forma escreve-se estoma, e não ostoma, como comumente é observado em alguns casos ${ }^{2}$.

A estomia tem como objetivo realizar as funções do órgão danificado a partir de procedimento cirúrgico no qual é realizada uma abertura para contato com o meio exterior ${ }^{3}$. A nomeação da estomia varia de acordo com o segmento corporal afetado. Assim, tem-se a traqueostomia, que e a abertura da traqueia; a estomia gástrica, denominada gastrostomia; as estomias urinárias, urostomias, que podem ser classificadas em nefrostomia, ureterostomia e cistostomia; e as estomias intestinais, que são as jejunostomias, ileostomias e colostomias ${ }^{4,5}$.

Independentemente de ser temporária ou definitiva, a realização desse procedimento acarreta uma série de mudanças na vida do paciente e requer um cuidado especializado de enfermagem. No que se refere à epidemiologia do tema em questão, destaca-se a inexistência de dados oficiais do Ministério da Saúde sobre a situação dos estomizados no Brasil.
Desse modo, segundo Vladimir Kleinwachter, vice-presidente da International Ostomy Association, há cerca de um estomizado para cada 1.000 habitantes, em países com um bom nível de assistência médica, podendo ser bem inferior nos países menos desenvolvidos ${ }^{6}$. Essa estimativa foi baseada em coleta de dados feita junto aos países membros da organização, constatando-se ausência de muitas respostas. Projetando-se essa estimativa para o nosso país tendo como base o censo de 2010 do Instituto Brasileiro de Geografia e Estatística $(\mathrm{IBGE})^{7}$, quando a população total era de 190.732 .694 pessoas, teríamos cerca de 190 mil estomizados.

Em publicação recente, na Revista da Associação Brasileira de Ostomizados (ABRASO), a respeito dos relatórios de alguns países exibidos na Conferência Anual da United Ostomy Association, no Canadá em 2005, têm-se: a Sérvia com 4.000 estomizados, clientela que ocupa o terceiro lugar no país, somente após diabéticos e hemofílicos; a Ucrânia, com 50 mil e a Rússia com 120 mil pessoas com estomas. Além disso, a associação japonesa contava, em 2005, com 12 mil membros, com média etária de 61 anos $^{8}$.

Frente a essa situação, o estomizado é amparado por um arcabouço legal que se fortalece ao longo dos anos, pois antes do ano de 2004 os direitos dos estomizados estavam basicamente, ou melhor, resumidamente, restritos ao fornecimento de bolsas coletoras, como previsto nas Portarias 116/939 e 146/93 ${ }^{10}$, do Ministério da Saúde.

Essa história ganha uma nova dimensão com a publicação da Lei 5296, de 2 de dezembro de 2004, cujo Artigo 5o (quinto) classifica os portadores de ostomia como deficientes físicos. Nesse novo contexto, os ostomizados passam a ter todos os direitos de uma Pessoa com Deficiência. Dessa 
forma, ganham o amparo de um significativo conjunto de leis, que regulamentam os direitos dos deficientes no Brasil, nas esferas federal, estadual e municipal ${ }^{11}$.

A condição de estomizado implica em mudanças no estilo de vida não só da pessoa com estoma, mas da sua família. Evidencia-se que o processo de reabilitação deve ser implementado com o paciente e sua família já na fase diagnóstica, visando restituir-lhe as atividades de convívio social e melhorar sua qualidade de vida diante do impacto de aquisição do estoma ${ }^{12}$. A família do estomizado conhece seus hábitos e preferências, ou seja, possui informações importantes que podem ser úteis no planejamento da reabilitação ${ }^{13}$.

A reabilitação e o autocuidado do cliente estomizado requerem do profissional enfermeiro atitudes de adequação da sua prática às necessidades dessa clientela, focadas na questão educativa. Assim, entende-se educação como um processo dinâmico, criativo, progressivo, reflexivo e libertador, contextualizada no universo cultural onde se compartilha o aprendizado, objetivando o aproveitamento máximo das capacidades residuais ou potencialidades do cliente ${ }^{14}$.

É de suma importância ressaltar nesse processo de ensino a Teoria do Autocuidado formalizada pela teórica Dorothea Orem, que permite subsídios para conscientização do paciente referente à importância de ser também responsável pelo seu cuidado ${ }^{15}$. A Teoria de Orem é baseada na premissa de que os pacientes podem cuidar de si mesmos, sendo encorajados a serem independentes o máximo possível, e quando um indivíduo é incapaz de cumprir seus requisitos de autocuidado, é papel do enfermeiro determinar esses déficit e definir as modalidades de suporte ${ }^{16}$.

A educação em saúde é considerada uma função inerente à prática de enfermagem e uma responsabilidade essencial da profissão ${ }^{17}$. Neste sentido, o enfermeiro é um profissional qualificado para exercer a atividade de educação em saúde, pois. além de obter conhecimento teórico. é a figura que está ao lado do enfermo durante a maior parte do período de internação.

Observa-se a necessidade de uma efetiva orientação para dar continuidade ao cuidado, satisfazendo as necessidades do paciente de forma eficaz e atendendo integralmente o indivíduo, sua família e a comunidade. Assim, cabe ao enfermeiro, nesta oportunidade, promover práticas educativas de modo que a família e o paciente sejam ouvidos, envolvidos e tranquilizados para a nova situação de vida.
A necessidade de elaboração deste estudo era evidente, visto que ao realizar o cuidado de enfermagem ao paciente estomizado identificava que muitos deles verbalizavam atitudes de autocuidado incorretas fornecidas por profissionais da saúde. Essa realidade foi o fator propulsor que despertou o interesse para realizar este estudo referente ás orientações de cuidado aos adultos estomizados.

A relevância deste estudo constitui-se pela contribuição a temática supracitada e pelo subsidio ao atendimento às pessoas estomizadas, fomentando assim a construção de uma visão mais ampliada por profissionais enfermeiros sobre orientações de cuidado ao paciente estomizado.

O objetivo desta pesquisa foi analisar evidências disponíveis quanto às orientações de enfermagem fornecidas a pessoa estomizada, caracterizando os elementos inerentes ao tema, bem como pontuar o conteúdo abordado e identificar estratégias utilizadas e o momento da orientação.

\section{MÉTODO}

Para a realização deste estudo, utilizou-se a revisão integrativa, por ser um método amplo, que busca aprofundamento sobre determinado assunto, a fim de promover a melhoria da assistência prestada ao paciente, embasada em evidências e fundamentada por estudos anteriores. Seguiram-se as seguintes etapas: formulação do problema; coleta, avaliação, análise e interpretação dos dados coletados e apresentação dos resultados ${ }^{18}$.

Para atender o objetivo desta pesquisa, este estudo teve como questão norteadora: como é realizada e quais são as orientações de enfermagem fornecidas à pessoa estomizada?

Os estudos selecionados seguiram os seguintes critérios de inclusão: estudos com métodos explícitos, publicados em português, realizados com adultos e idosos de ambos os sexos, em periódicos on-line gratuitos a partir da busca avançada. E, como critérios de exclusão: estudos duplicados e sem aderência ao objetivo do estudo.

O período da coleta de dados foi de junho a julho de 2014, nas bases de dados Literatura Latino-Americana e do Caribe em Ciências da Saúde (LILACS), Sistema Online de Busca e Análise de Literatura Médica (Medical Literature Analysis and Retrieval System Online - MEDLINE), a base de dados bibliográficos da Biblioteca Nacional de Medicina dos Estados Unidos da América, Base de Dados de Enfermagem da Biblioteca virtual em Saúde (BDENF/ 
BVS), Coordenação de Aperfeiçoamento de Pessoal de Nível Superior (CAPES), Scientific Electronic Library Online (SciELO). Optou-se por buscar estudos publicados nos últimos 10 anos, ou seja, de 2005 a 2014. Os Descritores em Ciências da Saúde (DECS) utilizados foram: estomia, educação em saúde e enfermagem.

A inclusão de estudos que foram encontrados por meio do descritor "estomia" quanto pelo cruzamento duplo "estomia + educação em saúde" e pelo cruzamento triplo "estomia + educação em saúde + enfermagem" ocorreu pelo fato de que alguns eram obtidos somente com o primeiro descritor e outros somente utilizando dois ou três descritores, ressalta-se que nos cruzamentos foram utilizados o operador Booleano "AND”. Obteve-se um total de 1.549 referências (Tabela 1) e, após a aplicação dos critérios de inclusão e exclusão anteriormente descritos, obteve-se uma amostra de 10 artigos.

Para coletar os dados, foi utilizado um instrumento com os seguintes quesitos: título autor, ano, revista, idade, tipo de estudo, momento orientação, tipo de estoma, estratégia, conteúdo.

\section{RESULTADOS}

A maioria dos periódicos (Quadro 1) é da área da enfermagem (Revista Brasileira de Enfermagem, Escola Anna Nery Revista de Enfermagem, Acta Paulista de Enfermagem, Texto e Contexto de Enfermagem e Perspectivas online de Enfermagem), sendo apenas dois da área da saúde (Recom e Revrene), não específicos para enfermagem, ressaltando que todos os autores são enfermeiros. Os trabalhos encontrados trazem em seus títulos a palavra cuidado, enfatizando as práticas realizadas para a manutenção de estomas por enfermeiros, familiares, cuidadores ou pelo próprio cliente.
Os temas apresentam diversas abordagens, dentre elas, a vivência da pessoa estomizada e do cuidador familiar, autocuidado, o idoso estomizado, reabilitação em enfermagem, educação permanente para enfermeiros e a educação da pessoa estomizada. Dessa forma, observa-se que os estudos abordam a pessoa estomizada em diferentes temas que convergem para o assunto em questão.

Dos 10 artigos que compuseram a amostra desta revisão integrativa, 5 (50\%) foram encontrados nas bases de dados LILACS, 4 (40\%) na BDENF e 1 (10\%) na CAPES.As publicações se concentram no período de 2009 a 2010 (50\%),30\% nos anos de 2012 a 2013 e 20\% 2007 a 2008 (Quadro 1).

A abordagem metodológica utilizada foi a qualitativa, para avaliar as orientações de enfermagem às pessoas estomizadas, entre eles predominou o estudo exploratório. Os autores que mais estudaram o tema em questão foram Martins e Alvim, com 30\% das publicações. Muitos autores realizam pesquisas focando as orientações de enfermagem a pessoa estomizada, porém poucos avaliam a efetivação das mesmas. Os tipos de estomias referidos nos estudos foram as intestinais e urinárias (Quadro 2).

Conforme descrito nos critérios de inclusão, esta revisão integrativa foca a avaliação das orientações de enfermagem direcionadas a adultos e idosos. Observa-se que cinco dos periódicos não especificaram a idade dos estomizados, definindo-os apenas como maior de 18 anos (N1); adulto e idosos (N5 a 7) e somente como adultos (N8), os demais que especificam as idades, dois realizado com idosos jovens (N4: 62 anos) e de mais idade/idosos mais velhos (N3: 93 anos); quanto aos adultos observa-se adultos jovens (N1: 26 anos, N2 e N9: 18 anos) e somente o artigo N4: 34 anos.

Ao analisar o momento que se iniciou esta orientação, observamos que somente um estudo enfatizou o início da prática das orientações de enfermagem no período pré-operatório, e os demais 90\% no pós-operatório (Quadro 2).

Tabela 1. Distribuição dos artigos científicos obtidos nas bases de dados: BDENF, Lilacs, Capes, Medline e SciELO, segundo Descritores em Ciências da Saúde.

\begin{tabular}{lcccccc}
\hline DECS & BDENF & LILACS & CAPES & MEDLINE & Scielo & Total \\
\hline Estomia & 84 & 186 & 40 & 1.057 & 21 & 1.388 \\
\hline Estomia AND educação em saúde & 8 & 13 & 24 & 45 & 1 & 91 \\
Estomia AND Educação em Saúde & 7 & 11 & 8 & 43 & 1 & 70 \\
AND Enfermagem & 99 & 210 & 72 & 1.145 & 23 & 1.549 \\
\hline Total & 99 & &
\end{tabular}

DECS: descritores em ciências da saúde; BDENF: BDENF: Base de dados de enfermagem; LILACS: Literatura Latino-Americana e do Caribe em Ciências da Saúde; CAPES: Coordenação de Aperfeiçoamento de Pessoal de Nível Superior; MEDLINE: Literatura Internacional em Ciências da Saúde. 
Quadro 1. Artigos publicados no período de 2005 a 2014, segundo título, autor, ano, periódico, idade e tipo de estudo.

\begin{tabular}{|c|c|c|c|c|c|}
\hline $\mathrm{N}$ & Título & Autor/ano & Periódico & Idade & Tipo de estudo \\
\hline 01 & $\begin{array}{c}\text { Vivências do cuidador familiar de } \\
\text { uma pessoa com estomia intestinal } \\
\text { por câncer colorretal. }\end{array}$ & $\begin{array}{l}\text { Oliveira et al. } \\
\qquad(2014)^{17}\end{array}$ & Revrene & 26 a 57 & $\begin{array}{l}\text { Exploratório } \\
\text { Descritivo } \\
\text { Qualitativo }\end{array}$ \\
\hline 02 & $\begin{array}{l}\text { Análise do autocuidado das pessoas } \\
\text { estomizadas em um município do } \\
\text { centro oeste de Minas gerais. }\end{array}$ & $\begin{array}{c}\text { Moraes, Sousa e } \\
\text { Carmo }(2012)^{22}\end{array}$ & Recom & 18 a 59 & $\begin{array}{l}\text { Transversal } \\
\text { Qualitativo }\end{array}$ \\
\hline 03 & $\begin{array}{l}\text { Ações ecossistêmicas e } \\
\text { gerontotecnológicas no cuidado } \\
\text { de enfermagem complexo ao } \\
\text { idoso estomizado. }\end{array}$ & $\begin{array}{c}\text { Barros et al. } \\
(2014)^{34}\end{array}$ & Rev Bras Enf. & 60 a 93 & $\begin{array}{l}\text { Qualitativo } \\
\text { Descritiva do tipo } \\
\text { estudo de caso }\end{array}$ \\
\hline 04 & $\begin{array}{l}\text { O enfermeiro e sua participação } \\
\text { no processo de reabilitação } \\
\text { da pessoa com estoma. }\end{array}$ & $\begin{array}{l}\text { Mauricio, Souza } \\
\text { e Lisboa }(2013)^{35}\end{array}$ & $\begin{array}{l}\text { Esc Anna Nery } \\
\text { Rev de Enf. }\end{array}$ & 35 a 62 & $\begin{array}{l}\text { Descritivo- } \\
\text { exploratório } \\
\text { Qualitativo }\end{array}$ \\
\hline 05 & $\begin{array}{l}\text { Desvelamento crítico da pessoa } \\
\text { estomizada: em ação o programa de } \\
\text { educação permanente em saúde. }\end{array}$ & $\begin{array}{l}\text { Cunha, Backes } \\
\text { e Heidemann } \\
(2012)^{36}\end{array}$ & Acta Paul Enf. & $\begin{array}{l}\text { Adulto } \\
\text { e Idoso }\end{array}$ & Qualitativo \\
\hline 06 & $\begin{array}{l}\text { Plano de cuidados compartilhado junto } \\
\text { a clientes estomizados: a pedagogia } \\
\text { freireana e suas contribuições à prática } \\
\text { educativa da enfermagem. }\end{array}$ & $\begin{array}{l}\text { Martins e Alvim } \\
\quad(2012)^{37}\end{array}$ & $\begin{array}{c}\text { Texto } \\
\text { Contexto Enf. }\end{array}$ & $\begin{array}{l}\text { Adulto } \\
\text { e idoso }\end{array}$ & $\begin{array}{l}\text { Convergente } \\
\text { Assistencial } \\
\text { Qualitativo }\end{array}$ \\
\hline 07 & $\begin{array}{l}\text { Saberes e práticas de clientes } \\
\text { estomizados sobre a manutenção } \\
\text { da estomia de eliminação intestinal e } \\
\text { urinária e sua pertinência no cuidado. }\end{array}$ & $\begin{array}{l}\text { Martins e Alvim } \\
\quad(2012)^{23}\end{array}$ & $\begin{array}{l}\text { Perspectivas } \\
\text { online }\end{array}$ & $\begin{array}{l}\text { Adultos } \\
\text { e Idoso }\end{array}$ & $\begin{array}{l}\text { Descritivo- } \\
\text { exploratório } \\
\text { Qualitativo }\end{array}$ \\
\hline 08 & $\begin{array}{l}\text { Perspectiva educativa do cuidado } \\
\text { de enfermagem sobre a manutenção } \\
\text { da estomia de eliminação. }\end{array}$ & $\begin{array}{l}\text { Martins e Alvim } \\
\quad(2011)^{21}\end{array}$ & Rev Bras Enf. & Adultos & $\begin{array}{l}\text { Convergente } \\
\text { Assistencial } \\
\text { Qualitativo }\end{array}$ \\
\hline 09 & $\begin{array}{c}\text { Vivência do paciente estomizado: } \\
\text { uma contribuição para a Assistência } \\
\text { de enfermagem. }\end{array}$ & $\begin{array}{l}\text { Nascimento } \\
\text { et al. }(2011)^{26}\end{array}$ & $\begin{array}{c}\text { Texto } \\
\text { Contexto Enf. }\end{array}$ & $>18$ & $\begin{array}{l}\text { Exploratório } \\
\text { Descritivo } \\
\text { Qualitativo }\end{array}$ \\
\hline 10 & $\begin{array}{l}\text { Prática de autocuidado de } \\
\text { estomizados: contribuições } \\
\text { da teoria de orem. }\end{array}$ & $\begin{array}{l}\text { Menezes et al. } \\
\qquad(2013)^{20}\end{array}$ & Rev Rene & 31 a 51 & $\begin{array}{l}\text { Descritiva } \\
\text { Qualitativo }\end{array}$ \\
\hline
\end{tabular}

Quadro 2. Distribuição dos artigos segundo o tipo de estoma, o momento e as estratégias das orientações.

\begin{tabular}{|c|c|c|c|c|}
\hline $\mathrm{N}$ & Autor/ Ano & $\begin{array}{l}\text { Tipo de } \\
\text { estoma }\end{array}$ & Momento & Estratégias \\
\hline 01 & Oliveira et al. (2014) $)^{17}$ & Intestinal & Pós op. & Verbal \\
\hline 02 & Moraes, Sousa e Carmo $(2012)^{22}$ & Intestinal & Pré e Pós op. & Verbal \\
\hline 03 & Barros et al. $(2014)^{34}$ & Intestinal & Pós op. & Verbal + demonstração em grupo \\
\hline 04 & Mauricio, Souza e Lisboa $(2013)^{35}$ & Intestinal & Pós op. & Verbal \\
\hline 05 & Cunha, Backes e Heidemann $(2012)^{36}$ & Intestinal & Pós op. & Círculo dialógico \\
\hline 06 & Martins e Alvim $(2012)^{37}$ & $\begin{array}{l}\text { Intestinal } \\
\text { Urinário }\end{array}$ & Pós op. & $\begin{array}{c}\text { Pedagogia problematizadora } \\
\text { Diálogo }\end{array}$ \\
\hline 07 & Martins e Alvim $(2012)^{23}$ & $\begin{array}{l}\text { Intestinal } \\
\text { Urinário }\end{array}$ & Pós op. & Verbal + treinamento \\
\hline 08 & Martins e Alvim (2011) $)^{21}$ & $\begin{array}{l}\text { Intestinal } \\
\text { Urinário }\end{array}$ & Pós op. & Prática dialógica \\
\hline 09 & Nascimento et al. $(2013)^{26}$ & Intestinal & Pós op. & Verbal \\
\hline 10 & Menezes et al. $(2013)^{20}$ & Intestinal & Pós op. & Verbal \\
\hline
\end{tabular}

op.: operatório 


\section{DISCUSSÃO}

Os dados coletados nesta revisão emergiram em três categorias: o momento da orientação, o tema abordado e as estratégias utilizadas.

\section{Momento da orientação}

Quando se fala em estomizado é de suma importância focar as orientações referentes aos cuidados que serão fornecidas ao paciente, e o momento que devemos orientá-los para promoção eficaz do autocuidado.

A literatura preconiza que o ensino do autocuidado deve ser iniciado logo após a decisão sobre o procedimento terapêutico a ser realizado. No período pré-operatório, logo no momento da admissão hospitalar, o paciente deve receber as principais orientações sobre sua futura condição de vida e os cuidados que, a partir de então, serão necessários. No pós-operatório imediato e tardio, o paciente deverá esclarecer suas dúvidas, demonstrar suas habilidades e mostrar-se capaz de realizar os cuidados domiciliares; caso seja necessário um cuidador, a demonstração será realizada por este. No momento da alta, o paciente será encaminhado ao Programa de assistência aos Ostomizados, para receber atendimento especializado e fornecimento dos equipamentos necessários à sua nova condição ${ }^{19-20}$.

A amostra N2 ressalta que a importância da orientação ser iniciada nesse momento deve-se ao fato de nesse período ser avaliado questões físicas (nutrição, funcionamento intestinal, comorbidades), questões sociais relacionadas à dinâmica familiar e de suporte emocional permitindo ao estomizado trabalhar sua estratégia de enfrentamento ${ }^{22}$. Nesse momento, o paciente absorve melhor as informações e isso pode contribuir para seu ajuste físico e psicológico após a cirurgia, uma melhor recuperação, adequação e sucesso no autocuidado. É ressaltado, também, que todas as informações pré-operatórias contribuem para o ensino pós-operatório, o qual deve ser contínuo e focado nas dificuldades dos pacientes e seus familiares/cuidadores ${ }^{19-21}$

Ao descrever um cuidado de enfermagem de qualidade fica evidenciado que, quando iniciado ainda no período pré-operatório, permite à pessoa uma orientação e preparação para lidar com esse novo modo de vida, favorecendo o desenvolvimento de habilidades necessárias e sanando as dúvidas referentes ao autocuidado que terá que realizar dali para frente ${ }^{23}$.
A enfermagem pode realizar suas orientações juntamente com a equipe multidisciplinar, na qual deve abordar o paciente e sua família com linguagem clara e de fácil entendimento, oferecendo orientações individualizadas na busca de atender às expectativas dos mesmos ${ }^{24}$.

A literatura corrobora com a afirmação anterior ao mencionar que a falta de uma assistência adequada, especialmente direcionada e articulada nas fases pré, trans e pós-operatório, envolvendo uma equipe multiprofissional, o próprio cliente e familiares, pode ocasionar dificuldade na aceitação da estomia, a adaptação dos novos hábitos de vida, além da rejeição da prática do autocuidado ${ }^{25}$.

\section{Conteúdo abordado nas orientações}

Ao analisar o conteúdo abordado nas orientações de enfermagem, constatou-se que o assunto mais discutido foi higienização e troca de bolsa, os assuntos eventualmente mencionados foram dieta, uso de adjuvantes, uso de acessórios, vestuário, vida social e familiar, atividade laboral e autoirrigação; e os assuntos menos abordados foram direitos e deveres, religiosidade e espiritualidade, complicações, busca de atendimento especializado e sexualidade.

É notório que assuntos como o conceito da própria estomia não foi abordado em nenhum artigo. Assim, compreende-se que a pessoa estomizada não recebe assistência de forma holística, mas, sim, fragmentada; deixando dúvidas a esclarecer. O conteúdo das orientações é variável entre os artigos não havendo nenhuma padronização ou consensos e diretrizes para o assunto a ser abordado.

O enfermeiro, ao oferecer orientações aos pacientes com estomia e aos seus familiares por meio de práticas educativas, pode dialogar sobre assuntos como sexualidade, aceitação da doença, superação da discriminação, prevenção de complicações relacionadas ao estoma e inserção no convívio social ${ }^{26,27}$.

Em contrapartida, Santos defende a ideia que o conteúdo do ensino é baseado naquilo que o paciente identifica como necessidade, a partir de observações feitas por ele mesmo, por sua família e também pelos profissionais de saúde. A enfermagem tem papel fundamental na recuperação e alcance de reabilitação do estomizado intestinal, pela característica do seu trabalho de assistência ao paciente que possibilita maior tempo de contato e estabelecimento de maior vínculo no ensino do paciente e de sua família, com abordagem de aspectos físicos e psicossociais, como dieta, controle do 
odor, manuseio e troca de equipamentos, autoestima, imagem corporal, utilização de roupas e sexualidade ${ }^{28}$.

\section{Estratégias utilizadas}

Dentre as estratégias utilizadas, observamos a predominância da verbal sobre a demonstração de grupo, círculo dialógico, prática dialógica, pedagogia problematizadora e treinamento. Não houve em nenhum artigo o uso de materiais impressos.

A técnica de utilização de materiais complementares com o objetivo de reforçar as informações verbais

tem sido descrita por educadores como a melhor forma de ensinar indivíduos adultos, visto que as pessoas retêm apenas $20 \%$ do que ouvem, $30 \%$ do que veem, $50 \%$ do que ouvem e veem, $70 \%$ do que ouvem, veem e dizem e $90 \%$ do que ouvem, veem, dizem e fazem ${ }^{29}$.

Um estudo ${ }^{30}$ explorou a produção bibliográfica acerca do ensino ao paciente no cuidado com o estoma, objetivando compreender o verdadeiro significado/valor do ensino aos pacientes na execução de suas práticas e habilidades no cuidado do estoma, e demonstrou que o aprendizado envolve habilidades (psicomotoras), conhecimento (cognitivo) e atitude (afetivo). Ressaltou a importância do enfermeiro no ensino das práticas de cuidado aos pacientes com estomas, pois as intervenções de enfermagem facilitam a adaptação e a realização de habilidades práticas do paciente, mas não há citação de uma estratégia de ensino específica para o ensino dos pacientes estomizados.

Outro estudo ${ }^{21}$ demonstrou a necessidade da avaliação do paciente com estomia pelo enfermeiro e descreveu como deve ser um ambiente adequado na hora da avaliação, como devem ser realizadas as orientações, a importância de reconhecer as necessidades de cada paciente, e contribuiu com um modelo descritivo das orientações de como o paciente deve retirar e colocar uma bolsa de estomia.

O conhecimento prévio por parte do enfermeiro sobre educação em saúde e sobre o processo de ensino aprendizagem é importante para identificar as dificuldades no aprendizado dos pacientes estomizados e selecionar métodos, técnicas e estratégias apropriados, de modo a facilitar o desenvolvimento do autocuidado, pois são os enfermeiros os responsáveis pela capacitação de sua equipe para cuidar desses pacientes. É ressaltado que a educação do paciente estomizado e seu familiar/cuidador deve ser gradual, para que possam assimilar os dados e assimilar formações recebidas, atender às expectativas, esclarecer dúvidas e diminuir a ansiedade do indivíduo frente ao ato cirúrgico ${ }^{31}$.

Ensinar pacientes com uma estomia é um processo complexo, que exige avaliação prévia, planejamento e treinamento dos cuidadores, portanto, a aprendizagem depende de três domínios: cognitivo, afetivo e psicomotor ${ }^{32}$. Os desafios para uma instrução eficaz são: necessidade do tempo limitado para o ensino, e diminuição de custos e necessidade de ensinar populações cada vez mais diversas em fase de envelhecimento. Não obstante, a aplicação de princípios bem conhecidos de ensino e aprendizagem pode tornar o processo eficiente, eficaz e satisfatório ao paciente e ao enfermeiro ${ }^{33}$.

\section{CONCLUSÃO}

Diante do exposto, constatamos que a abordagem temática "cuidado ao estomizado" na qual está inserida as orientações de enfermagem é muito discutida. Porém, poucos estudos analisam os resultados das mesmas, isto é, se de fato atendem as necessidades da pessoa estomizada ou se abordam o conteúdo necessário para o cuidado adequado com o estoma.

Concluímos que o inicio tardio destas orientações de enfermagem dificultam a adaptação a essa nova realidade prolongando o período de reabilitação e causando impacto aos pacientes e seus familiares nos cuidados a serem realizados após a alta hospitalar e que o uso de estratégias complementares a verbal favorecem a aquisição de aprendizagem, visto que as pessoas retêm mais conteúdo quando ouvem, veem, dizem e fazem. $\mathrm{O}$ conteúdo deve ser abordado de forma holística que contemple informações que vão além da higienização e da troca da bolsa, pois para fortalecer a prática educativa de enfermagem é necessário assistência individual, integral e sistematizada ao estomizado

Estes dados evidenciaram uma imensa lacuna no processo de reabilitação da pessoa com estoma e que podem ser solucionados com resgate das orientações fornecidas à clientela em termos de apreensão das informações; reconhecimento do impacto da presença da estomia na pessoa idosa; promoção de dinâmicas interativas entre os pacientes que favoreçam a troca de vivências e experiências; elaboração de tecnologias educativas de assistência perioperatória como cartilhas e manuais e sistematização da assistência de enfermagem. 


\section{REFERÊNCIAS}

1. Santos VLCG. A bolsa na mediação "estar ostomizado" e "estar profissional": análise de uma estratégia pedagógica [tese]. São Paulo: Universidade de São Paulo; 1996.

2. Bacelar S, Galvão CC, Tubino P. Expressões médicas errôneas: erros e acertos. Acta Cir Bras. 2004;19(5):582-3.

3. Gemelli LMG, Zago MMF. A interpretação do cuidado com o ostomizado na visão do enfermeiro: um estudo de caso. Rev Lat Am Enferm. 2002;10(1):34-40.

4. Martins Jr A, Rocha JJR. Tipos de estoma. In: Crema E, Silva R. Estomas: uma abordagem interdisciplinar. Uberaba: Pinti; 1997. p. 43-4.

5. Santos VLCG. Estomaterapia através dos tempos. In: Santos VLCG, Cesaretti IUR. Assistência em estomaterapia cuidado do ostomizado. São Paulo: Atheneu; 2005. p. 1-17.

6. Santos VLCG. Aspectos epidemiológicos dos estomas. Rev Estima. 2007;5(1):31-8.

7. Instituto Brasileiro de Geografia e Estatística. Censo demográfico 2010. Rio de Janeiro; 2011 Disponível em: http://www.censo2010.ibge.gov.br. Acessado em: dezembro de 2014

8. Associação Brasileira de Ostomizados (ABRASO). Giro pelo mundo: ações pelo mundo. Rev Abraso. 2005;(5):19-21.

9. Brasil. Ministério da Saúde. Portaria $n^{\circ} 116$, de 9 de setembro de 1993. Inclui no Sistema de Informações Ambulatoriais do Sistema Único de Saúde - SIA/SUS a concessão dos equipamentos de órteses, próteses e bolsas de colostomia constantes do Anexo Único. Disponível em: http://bvsms.saude.gov.br/bvs/saudelegis/ sas/1993/prt0116_09_09_1993.html. Acessado em: dezembro de 2014

10. Brasil. Ministério da Saúde. Portaria n 146, de 14 de outubro de 1993. Estabelece diretrizes gerais para a concessão de Próteses e Órteses através da Assistência. Disponível em: http://bvsms.saude.gov.br/bvs/saudelegis/sas/1993/ prt0146_14_10_1993.html Acessado em: dezembro de 2014

11. Ostomizados \& Cia. Direitos dos ostomizados. Disponível em: http://www.ostomizadosecia.com/2010/05/direitos-dosostomizados.html. Acessado em: dezembro de 2014.

12. Bechara RN, Bechara MS, Bechara CS, Queiroz HC, Oliveira RB, Mota RS, et al. Abordagem multidisciplinar do ostomizado. Rev Bras Coloproct. 2005;25(2):146-9.

13. Menezes APS, Quintana JF. A percepção do indivíduo estomizado quanto à sua situação. Rev Bras Promoção Saúde. 2008;21(1):13-8.

14. Poggetto MT, Casagrande LDR. "Fui fazendo e aprendendo...": temática de aprendizagem de clientes colostomizados e a ação educativa do enfermeiro. Rev Min Enferm. 2003;7(1):28-34.

15. Leopardi, MT. Teorias de Enfermagem: instrumentos para a prática. Florianópolis: NFR/UFSC; 1999.

16. George JB. Teorias de enfermagem: os fundamentos para a prática profissional. Porto Alegre: Artes Médicas; 1993.

17. Oliveira GS, Bavaresco M, Filipini CB, Rosado SR, Dázio EMR, Fava SMCL. Vivências do cuidador familiar de uma pessoa com estomia intestinal por câncer colorretal. Rev Rene. 2014;15(1):108-15.

18. Mendes KDS, Silveira RCCP, Galvão CM. Revisão integrativa: método de pesquisa para a incorporação de evidências na saúde e na enfermagem. Texto Contexto Enferm. 2008;17(4):758-64.
19. Coey L. Readability of printed educational materials used to inform potential and actual ostomates. J Clin Nurs. 1996;5(6):359-66.

20. Menezes LCG, Guedes MVC, Oliveira RM, Oliveira SKP, Meneses LST, Castro ME. Prática de autocuidado de estomizados: contribuições da teoria de orem. Rev Rene. 2013;14(2):301-10.

21. Martins PAF, Alvim NAT. PP Perspectiva educativa do cuidado de enfermagem sobre a manutenção da estomia de eliminação. Rev Bras Enf. 2011;64(2):322-7.

22. Moraes JT, Sousa LA, Carmo WJ. Análise do autocuidado das pessoas estomizadas em um município do centro-oeste de Minas Gerais. Rev Enferm Cent-Oeste. Min. 2012;2(3):337-46.

23. Martins PAF, Alvim NAT. Saberes e práticas de clientes estomizados sobre a manutenção da estomia de eliminação intestinal e urinária e sua pertinência no cuidado. Perspectiva Online: biol \& saúde. 2012;6(2):54-69.

24. Barnabe NC, Dell'Acqua MCQ. Estratégias de enfrentamento (coping) de pessoas estomizadas. Revista Lat Am Enferm. 2008;16(4):712-9.

25. Santos VLCG, Sawaia BB. A bolsa na medição "estar ostomizado"-"estar profissional" análise de uma estratégia pedagógica. Rev Lat Am Enf. 2000;8(3):40-50.

26. Nascimento CMS, Trindade GLB, Luz MHBA, Santiago RF. Vivência do paciente estomizado: Uma contribuição para a assistência de enfermagem. Texto Contexto Enferm. 2011;20(3):357-64.

27. Santana JCBS, Dutra BS, Tameirão MA, Silva PF, Moura IC, Campos ACV. O significado de ser colostomizado e participar de um programa de atendimento ao ostomizado. Cogitare Enferm. 2010;15(4):631-8

28. Santos Jr JCM. O paciente cirúrgico idoso. Rev Bras Coloproctol. 2003;23(4):305-16.

29. Ducci AJ, Pimenta CAM. Programas educativos e a dor oncológica: estudo de revisão. Rev Bras Cancerol. 2003;49(3):185-92.

30. Metcalf C. Stoma care: empowering patients through teaching practical skills. Br J Nurs. 1999;8(9):593-600.

31. Santos GS, Leal SMC, Vargas MA. Conhecendo as vivências de mulheres ostomizadas: contribuições para o planejamento do cuidado de enfermagem. Online Braz J Nurs. 2006;5(1).

32. O'Shea HS. Teaching the adult ostomy patient. J Wound Ostomy Continence Nurs. 2001;28(1):47-54.

33. Cesaretti IUR, Vianna LAC. Sistema oclusor ou oclusor intermitente da colostomia: alternativa para a reabilitação da pessoa colostomizada. Acta Paul Enferm. 2003;16(3):98-108.

34. Barros EJL, Santos SSC, Gomes GC, Erdmann AL, Pelzer MT, Gautério DP. Ações ecossistêmicas e gerontotecnológicas no cuidado de enfermagem complexo ao idoso estomizado. Rev Bras Enferm. 2014;67(1):91-6.

35. Mauricio VC, Souza NVDO, Lisboa MTL. O enfermeiro e sua participação no processo de reabilitação da pessoa com estoma. Esc Anna Nery. 2013;17(3):416-22.

36. Cunha RR, Backes VMS, Heidemann ITSB. Desvelamento crítico da pessoa estomizada: em ação o programa de educação permanente em saúde. Acta Paul Enferm. 2012;25(2):296-301.

37. Martins PAF, Alvim NAT. Plano de cuidados compartilhado junto a clientes estomizados: a pedagogia freireana e suas contribuições à prática educativa da enfermagem. Texto Contexto Enferm. 2012;21(2):286-94. 